\title{
RASTRILLADAS DE ICNITAS TERÓPODAS GIGANTES DEL JURÁSICO SUPERIOR (SINCLINAL DE IOUARIDÈNE, MARRUECOS)
}

\author{
Mohamed BOUTAKIOUT ${ }^{1}$, Majid HADRI ${ }^{1}$, Jaouad \\ NOURI ${ }^{1}$, Ignacio DÍAZ-MARTÍNEZ ${ }^{2}$ y Félix \\ PÉREZ-LORENTE ${ }^{2}$
}

${ }^{1}$ Departement des Sciences de la Terre. Université Mohamed V. Av. Ibn Batota. Rabat-Agdal.Marruecos.boutak@fsr.ac.ma; hadri@fsr.ac.ma; jnouri1@yahoo.fr. ${ }^{2}$ Edificio CT. Universidad de La Rioja. Madre de Dios, 51-53. 26006 Logroño. España.inaportu@hotmail.com; felix.perez@unirioja.es

Boutakiout, M., Hadri, M., Nouri, J., Díaz-Martínez, I. \& Pérez-Lorente, F. 2009. Rastrilladas de icnitas terópodas gigantes del Jurásico Superior (Sinclinal de Iouaridène, Marruecos). [Gigantic theropod footprints from Upper Jurassic trackways (Iouaridène syncline, Morocco).] Revista Española de Paleontología, 24 (1), 31-46. ISSN 0213-6937.

\begin{abstract}
The dinosaur ichnological research in the Upper Jurassic lacustrine sediments of the Iouaridène syncline provide data of interest. One of them includes the discovery of gigantic digitigrade theropod footprints, with a not described size. The exposures of ichnitic levels are continuous in the zone and the continuity of stratigraphic beds permits the good correlation. All sites are directly linked, except one of them that are separated by a cover of quaternary glacis sediments. Inside the work area, that includes the half of the syncline, the giant footprints occupy a limited section that extends in the same direction of these footprints. The spatial situation allows us to propose that the gigantic footprints of every bed belong to the same trackway, with a maximum length $2000 \mathrm{~m}$. The direct and indirect footprint structures are distinctive from each to other trackway. However these are not the only footprints discovered; between and inside the sites, there are many smaller theropod and some ornithopod and sauropod prints. This shows that, at least three giant theropod dinosaurs lived in the same site with the smaller theropods being the last most prominent. After a comparative study it is proposed that two of the three gigantic theropod trackways and the digitigrade footprints described in this paper, are the largest discovered yet in the world.
\end{abstract}

Key words: Footprints, theropod, gigantism, Upper Jurassic, Hight Central Atlas, Morocco.

\section{RESUMEN}

La investigación sobre huellas de dinosaurio en rocas de origen lacustre del Jurásico Superior del sinclinal de Iouaridène está proporcionando datos de gran interés. Uno de ellos es el hallazgo de icnitas digitígradas terópodas gigantes, de un tamaño no descrito hasta el momento. Las capas donde están registradas las icnitas son contínuas y perfectamente identificables en el campo. Todos los yacimientos estudiados se pueden correlacionar directamente, excepto uno muy alejado, separado de los demás por recubrimiento de sedimentos de un glacis cuaternario. Dentro de toda la zona prospectada que abarca la mitad del sinclinal, las icnitas gigantes ocupan un sector delimitado y alargado cuya dirección coincide con la orientación de las huellas. Esta disposición espacial permite proponer la hipótesis de que las icnitas gigantes de una de las capas corresponderían a una rastrillada, que sería de una longitud máxima de $2.000 \mathrm{~m}$; por otro lado, las estructuras directas e indirectas son diferentes de una rastrillada a otra, lo que permite diferenciarlas. Además de las icnitas gigantes, en las capas también se han registrado numerosas icnitas terópodas más pequeñas, así como algunas ornitópodas y saurópodas. Se interpreta que pudo haber al menos tres dinosaurios terópodos gigantes en un paraje en el que predominaban terópodos más pequeños. Después del estudio comparativo se propone que dos de las tres rastrilladas de huellas gigantes, deben ser las más grandes descubiertas que existen actualmente en el mundo.

Palabras clave: Huellas fósiles, terópodo, gigantismo, Jurásico Superior, Alto Atlas Central, Marruecos. 


\section{INTRODUCCIÓN}

En el sinclinal de Iouaridène (Fig. 1) se ha realizado un primer trabajo de prospección por Boutakiout et al. (2009) en el que se citan 31 yacimientos con presencia de icnitas de dinosaurios (Fig. 2), que se distribuyen por la mitad occidental del sinclinal. Todos ellos están registrados en la Formación de Iouaridène y, dentro de ella, en los niveles de chert de color rojo más competentes, en los que son habituales las grietas de desecación y rizaduras. Se han reconocido distintos tipos de icnitas, distribuidas sin una ordenación aparente:

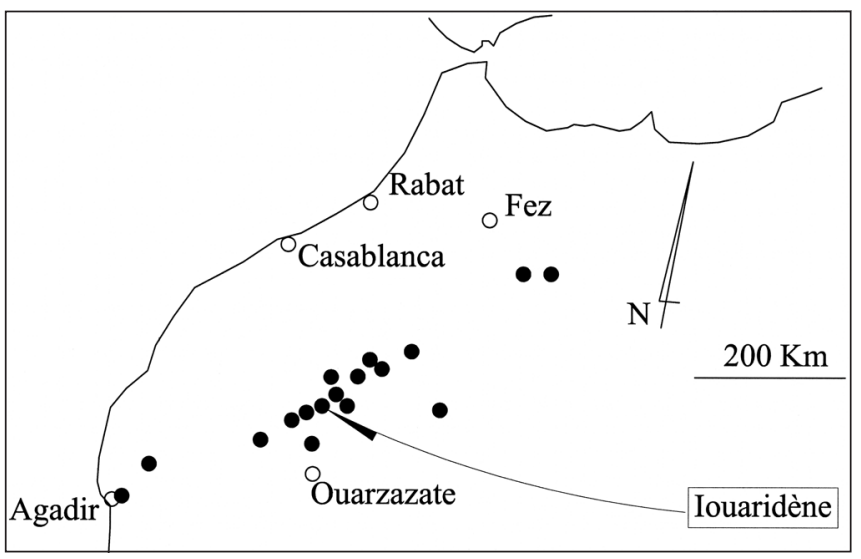

Figura 1. Situación geográfica de los yacimientos de icnitas de dinosaurios de Marruecos (Pérez-Lorente et al., 2006). Geographic situation of palaeoichnological sites of Morocco (Pérez-Lorente et al., 2006).
- abundantes icnitas tridáctilas y tetradáctilas (con hallux) terópodas;

- algunas ornitópodas de tamaño variable (llegan incluso a medir hasta $80 \mathrm{~cm}$ ); y

- saurópodas dispuestas en todas las direcciones del espacio.

Los 31 yacimientos con icnitas catalogados en el sinclinal se localizan en un número no determinado de capas de chert de la Formación de Iouaridène (Charrière et al., 2005), que coronan las capas lutíticas, de color ladrillo y rojo oscuro intenso (Nouri, 2007) de esta formación; estas lutitas no muestran estructuras internas visibles.

Los niveles de chert (silcretas), que resaltan en el paisaje por su dureza y mayor resistencia a la erosión, tienen una composición del 75-80 \% de sílice microcristalina (Caro, com pers.); son niveles centimétricos en los que se observan rizaduras, grietas de desecación y huellas de dinosaurio, separados o no entre ellos por niveles milimétricos de limolitas.

La composición litológica y las estructuras sedimentarias indican que la sedimentación de estas secuencias de la Formación Iouaridène se produjo en un ambiente lacustre con evaporación intensa del agua.

En el Alto Atlas central y oriental y en el Atlas Medio se han definido varias formaciones de edad Jurásico Medio a Cretácico Medio que se han denominado tradicionalmente con el nombre de "capas rojas" (Charrière et al., 2005). En el Alto Atlas central las capas rojas se dividen en tres formaciones:

- la Formación Guettioua, con areniscas gruesas;

- la Formación Iouaridène, con sedimentos detríticos más finos; y

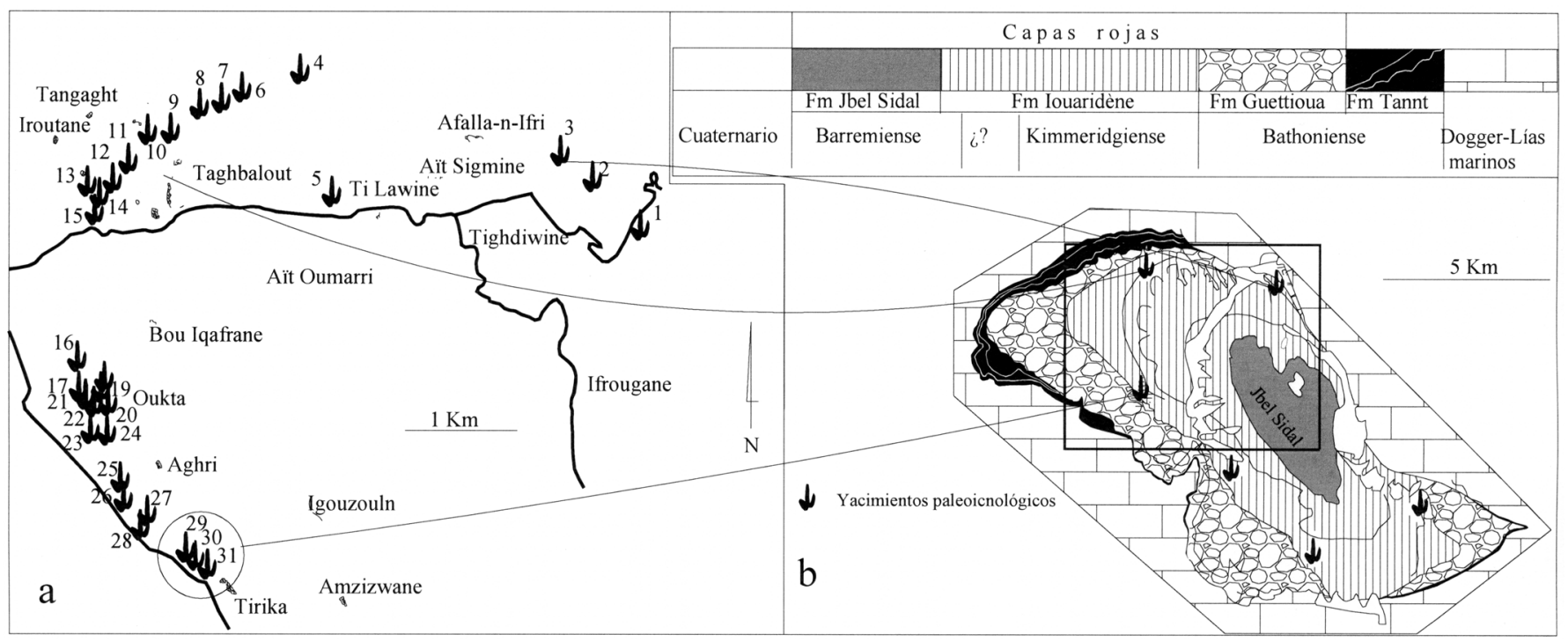

Figura 2. Sinclinal de Iouaridène. a, Situación de los yacimientos del sector Oeste (tomado de Boutakiout et al., 2009). b, Síntesis geológica a partir de Jenny (1982) y Charriere et al. (2005); yacimientos según Jenny (1985).

Iouaridène syncline. a,West ichnological site situation (after Boutakiout et al., 2009). b, Geological synthesis; data from Jenny (1982) and Charriere et al. (2005); ichnological sites after Jenny (1985). 
- la Formación Jebel Sidal, constituida en su mayor parte por areniscas.

Los materiales sedimentarios, según los mismos autores, se asignan a posiciones estratigráficas variadas que van desde el Jurásico Medio (Bathoniense?-Calloviense) hasta el Cretácico Inferior. La edad de la Formación de Iouaridène es Jurásico Superior y, concretamente, la parte en la que se registran las icnitas se ha datado con una edad Oxfordiense-Kimmeridgiense (Charrière et al., 2005).

En ocho de los afloramientos meridionales del sinclinal, se descubrieron pisadas terópodas tridáctilas gigantes de más de $70 \mathrm{~cm}$ de longitud en pequeños rastros que están orientados de forma paralela. Aunque no se ha terminado la prospección de la cubeta, es destacable que las icnitas gigantes posean una orientación subparalela y alineada entre sí en cada uno de los tres niveles donde se encuentran, según la posición de los afloramientos, por ello se propone que en cada uno de ellos pudiera registrarse una rastrillada.

La hipótesis de trabajo es que las pisadas terópodas gigantes formar parte de tres rastros (dos de ellos muy largos) impresos en tres niveles estratigráficos distintos, las icnitas se van a describir en consonancia con esta hipótesis. También, se comparan con otras rastrilladas y huellas de características similares del registro icnológico mundial.

Las capas con las icnitas gigantes tienen de dirección aproximada N-S y buzan hacia el Este menos de 15 grados. A pesar de la poca potencia de los niveles de chert, su continuidad es grande (Fig. 3) y se pueden seguir durante cientos de metros sin que estén rotos por fallas (Boutakiout et al., 2009). En estos niveles, además de las tres

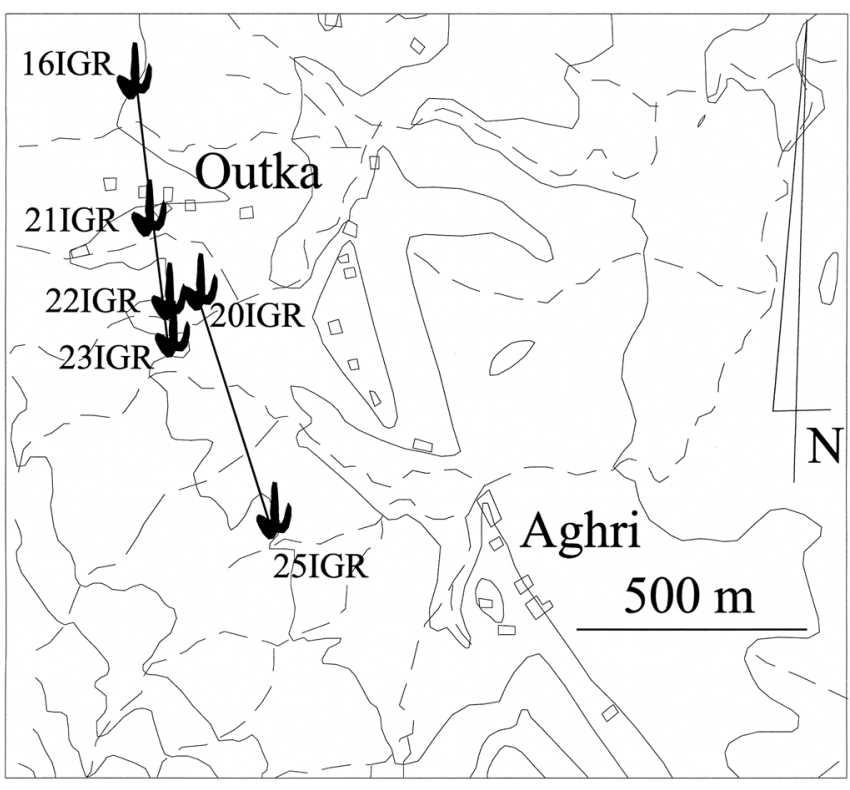

Figura 3. Situación detallada y trazado de los yacimientos de la rastrillada inferior (parte) y de la intermedia. A detailed situation and route of lower trackway (part) and middle trackway sites. rastrilladas aquí propuestas, también se encuentran algunas huellas de gran tamaño, como algunas icnitas ornitópodas, algunas pisadas de saurópodos de un metro de dimensión mayor (Nouri, 2007), así como, gran cantidad de huellas terópodas tridáctilas que no superan los $65 \mathrm{~cm}$ de largo.

Los ocho yacimientos estudiados (13IGR, 16IGR, 19IGR, 20IGR, 21IGR, 22IGR, 23IGR y 25IGR) han sido previamente citados por Boutakiout et al. (2008) con la misma numeración (Fig. 1, yacimientos: 13, 16, 19, 20, $21,22,23$ y 25). Las coordenadas UTM de las localidades (tabla 1) se han obtenido con un GPS Garmin, GPSMAP-60C. Los puntos se han situado en el campo sobre la Carte de Maroc 1:50.000 Feuille NH-29-XXIV-3a TIFNI.

\section{ESTUDIO ICNÍTICO}

Previamente a este trabajo se han descrito y/o citado distintos icnotipos e icnopoyetas citados en la región, que se comentan brevemente a continuación:

- Plateau et al. (1937) fueron los primeros que notificaron la presencia de huellas de dinosaurio en este sinclinal; señalan la presencia de icnitas tridáctilas de unos $50 \mathrm{~cm}$ de longitud, sobre su posible productor dicen (Op. cit.: 242): “ ... L. Joleaud ... propose de les rapprocher de celles que laisserait sur le sol un animal apparenté au Megalosaures, ..." ;

- Lapparent (1945: 270) comenta que "Les traces de Demnat, si on voulait les rapprocher de quelque chose de connu, devraient être rapportées au type 'Eubrontes' (= ¿Brontozoum') ...";

- Dutuit \& Ouazzou (1980) definen en este sinclinal (Fig. 2, yacimiento 9) Breviparopus taghbaloutensis, saurópodo de vía estrecha que contiene 32 pares mano-pie;

- Jenny et al. (1981: 428) cita huellas muy abundantes y "deux enigmatiques sillons parallèles (traces d'appendice caudal?)";

- Meyer \& Monbaron (2002) asignan varias huellas de pequeños terópodos halladas en las áreas de Demnat e Isseksi al icnogénero Carmelopus Lockley, Hunt, Paquette, Bilbey \& Hamblin, 1998;

- Recientemente, se ha definido en este sinclinal una icnita tetradáctila terópoda (Fig. 8, yacimiento 8) Eutynichnium atlasipodus Nouri, 2007. Este mismo autor asigna a icnitas terópodas el resto de las tridáctilas y tetradáctilas con dedos largos y acuminados que describe en su tesis (Nouri, op. cit.)

Las icnitas en este trabajo se han estudiado utilizando la caracterización biomórfica de acuerdo con los criterios propuestos por Romero et al. (2003) y la morfométrica siguiendo a varios autores (Alexander, 1976; Demathieu, 1986; Thulborm, 1990; Pérez-Lorente, 2001). Para denominar las estructuras que acompañan a las huellas se ha adoptado la terminología propuesta por Allen (1997), Fornós et al. (2002), Gatesy (2003), Requeta et al. (2006-7) y Pérez-Lorente \& Herrero Gascón (2007). 
La identifiación de las icnitas se hace de acuerdo con la nomenclatura habitual del grupo de trabajo y consta de cuatro partes:

- el primer número y las letras que le siguen indican el yacimiento;

- el segundo número determina la rastrillada y sirve para distinguirla de las que se encuentran en la misma localidad; $\mathrm{y}$

- el último número, separado por un punto, es el número de orden de la icnita.

Como ejemplo la sigla 25IGR1.1 indica que es la primera icnita del rastro 1 del yacimiento 25 de Iouaridène.

\section{DESCRIPCIÓN GENERAL (Figs. 2 y 4; Tabla 1)}

Las icnitas están agrupadas en conjuntos de dos o tres pisadas excepto para los ejemplares 13IGR1 (una) y en 25 IGR1 (siete) (Fig. 4). Todas ellas se ubican en tres niveles de chert, claramente identificables en el campo, sólo la icnita 13IGR1, presenta alguna duda, ya que está separada del resto de los afloramientos por recubrimiento cuaternario. Las icnitas reconocidas en cada capa son:

- capa inferior se localizan los grupos 16IGR1, 21IGR1, 22IGR1 y 23IGR1;

- capa intermedia 20IGR1 y 25IGR1; y

- capa superior 19IGR2.

Los grupos de icnitas terópodas grandes están orientadas todas entre N147E y N175E y su sentido de marcha es siempre hacia el Sur (Figs. 3 y 4), mientras que el resto de icnitas van en todas las direcciones y se registran por toda la zona prospectada del sinclinal. De acuerdo con la hipótesis de que estas huellas se agrupan en tres rastrilladas, los grandes terópodos deberían caminar solos por los mismos lugares que los deambulaban los demás dinosaurios de la región, de los que todavía no se ha hecho un análisis - ni taxonómico, ni etológico.

Las estructuras singenéticas relacionadas con la formación de las icnitas son diferentes para cada una de las rastrilladas, como se describe a continuación, pero son similares en todas las huellas que forman parte de la misma rastrillada o, lo que es lo mismo, en las huellas gigantes registradas en cada una de las capas. En todas las capas las grietas y polígonos de desecación están rotos, deformados y desplazados por las icnitas gigantes, por lo que dichas estructuras son anteriores al paso de los dinosaurios.

Rastrillada de la capa inferior (Figs. 2, 3, 4 y 5; Tablas 1 y 2)

Está constituida por once icnitas repartidas en cinco afloramientos. La distancia entre el primer afloramiento (13IGR1) y el último (23IGR1) es de 2.000 metros (Fig. 2). Los yacimientos más próximos (16IGR, 21IGR, 22IGR y 23 IGR) están en una línea que ocupa 555 metros (507 según las medidas de GPS).

La longitud media de las pisadas (Tablas 1 y 2) es de 75,5 cm (máximo de $80 \mathrm{~cm}$ en 16IGR1.1; mínimo de 70 cm en 16IGR1.4 y 22IGR1.2). La anchura media es de $60 \mathrm{~cm}$ (máximo de $76 \mathrm{~cm}$ en 16IGR1.1; mínimo de $53 \mathrm{~cm}$ 13IGR1.1).

No todas las pisadas presentan bien definidas las estructuras directas (almohadillas dactilares, «talón», etc), porque los techos de las capas presentaban antes del paso del dinosaurio grietas de desecación, que ahora se observan más o menos rotas en el fondo de las huellas.

Los dedos son gruesos, largos e independientes, de terminación acuminada y con marcas de almohadillas (Figs. 6 y 7). En las que se distingue bien el borde posterior («talón») se observa que es bilobulado, o con escotadura en la parte proximal del dedo II. El «talón» lo produce el dedo IV. El ángulo interdigital III^IV es mayor $\left(29^{\circ}\right)$ que el II^III $\left(15^{\circ}\right)$.

\begin{tabular}{|c|c|c|c|c|c|c|c|c|}
\hline rastrillada & 13IGR & 16IGR & 19IGR & 20IGR & 21IGR & 22IGR & 23IGR & 25IGR \\
\hline Y-UTM & 0698265 & 0698038 & 0698101 & 0698177 & 0698107 & 0698164 & 0698177 & 0698259 \\
\hline X-UTM & 3512435 & 3511160 & 3510748 & 3510737 & 3510951 & 3510729 & 3510672 & 3510166 \\
\hline profundidad & 6 & relleno & & 5 & & 12 & 7 & 4 \\
\hline Orientación & N147 & N175 & N158 & $\mathrm{N} 152$ & N170 & N155 & N174 & N157 \\
\hline Capa & ¿inferior? & inferior & superior & intermedia & inferior & inferior & inferior & intermedia \\
\hline $\begin{array}{l}\text { estructuras } \\
\text { sedimentarias }\end{array}$ & $\begin{array}{l}\text { rizaduras y grietas } \\
\text { de desecación }\end{array}$ & \begin{tabular}{|l} 
rizaduras y grietas \\
de desecación
\end{tabular} & \begin{tabular}{|l|} 
grietas de \\
desecacion \\
\end{tabular} & $\begin{array}{l}\text { rizaduras y grietas } \\
\text { de desecación }\end{array}$ & \begin{tabular}{|l} 
rizaduras y grietas \\
de desecación
\end{tabular} & $\begin{array}{l}\text { rizaduras y grietas } \\
\text { de desecación }\end{array}$ & $\begin{array}{l}\text { rizaduras y grietas } \\
\text { de desecación }\end{array}$ & $\begin{array}{l}\text { rizaduras y grietas } \\
\text { de desecación }\end{array}$ \\
\hline estructuras directas & $\begin{array}{l}\text { marcas de } \\
\text { almohadillas }\end{array}$ & \begin{tabular}{|l} 
polígonos \\
fracturados
\end{tabular} & flujo de barro & & \begin{tabular}{|l} 
polígonos \\
fracturados
\end{tabular} & \begin{tabular}{|l} 
polígonos \\
fracturados
\end{tabular} & $\begin{array}{l}\text { polígonos } \\
\text { fracturados } \\
\end{array}$ & \\
\hline otras estructuras & $\begin{array}{l}\text { polígonos } \\
\text { desplazados }\end{array}$ & \begin{tabular}{|l|} 
polígonos \\
desplazados y \\
doblados \\
\end{tabular} & $\begin{array}{l}\text { polígonos } \\
\text { desplazados }\end{array}$ & rebabas suaves & \begin{tabular}{|l|} 
polígonos \\
desplazados y \\
doblados \\
\end{tabular} & \begin{tabular}{|l|} 
polígonos \\
desplazados y \\
doblados \\
\end{tabular} & $\begin{array}{l}\text { polígonos } \\
\text { desplazados y } \\
\text { doblados } \\
\end{array}$ & rebabas suaves \\
\hline $1 / a$ & $79 / 53$ & $75 / 65$ & 90/-- & $80 / 66$ & $79 / 66$ & $70 / 55$ & $74 / 62$ & $73 / 64$ \\
\hline$h$ & 331 & 337 & 394 & 336 & 353 & 318 & 313 & 338 \\
\hline
\end{tabular}

Tabla 1. Características generales de las rastrilladas. Medidas en $\mathrm{cm}$. General characteristics of the trackways. Size in $\mathrm{cm}$. 


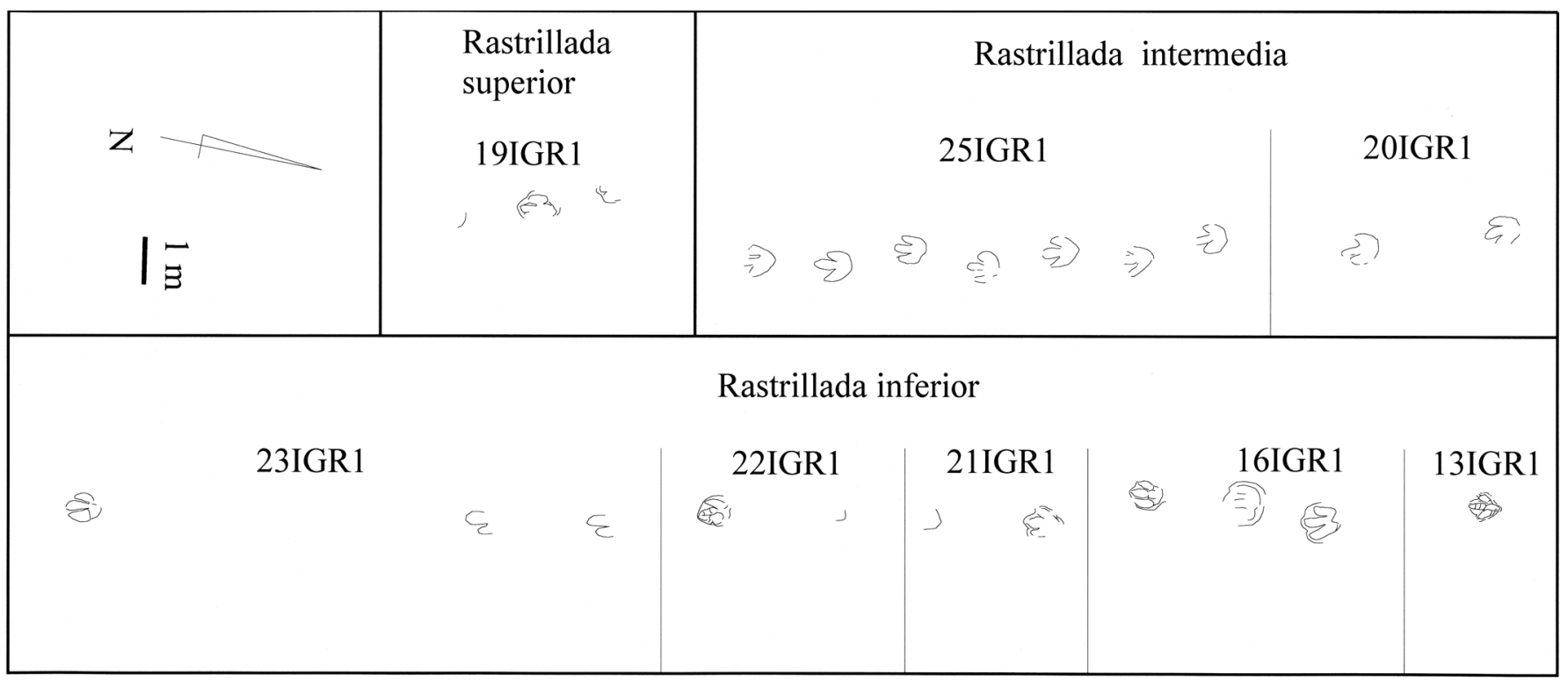

Figura 4. Las tres rastrilladas terópodas gigantes de Iouaridène. The three Iouaridène's gigantic theropod trackways.
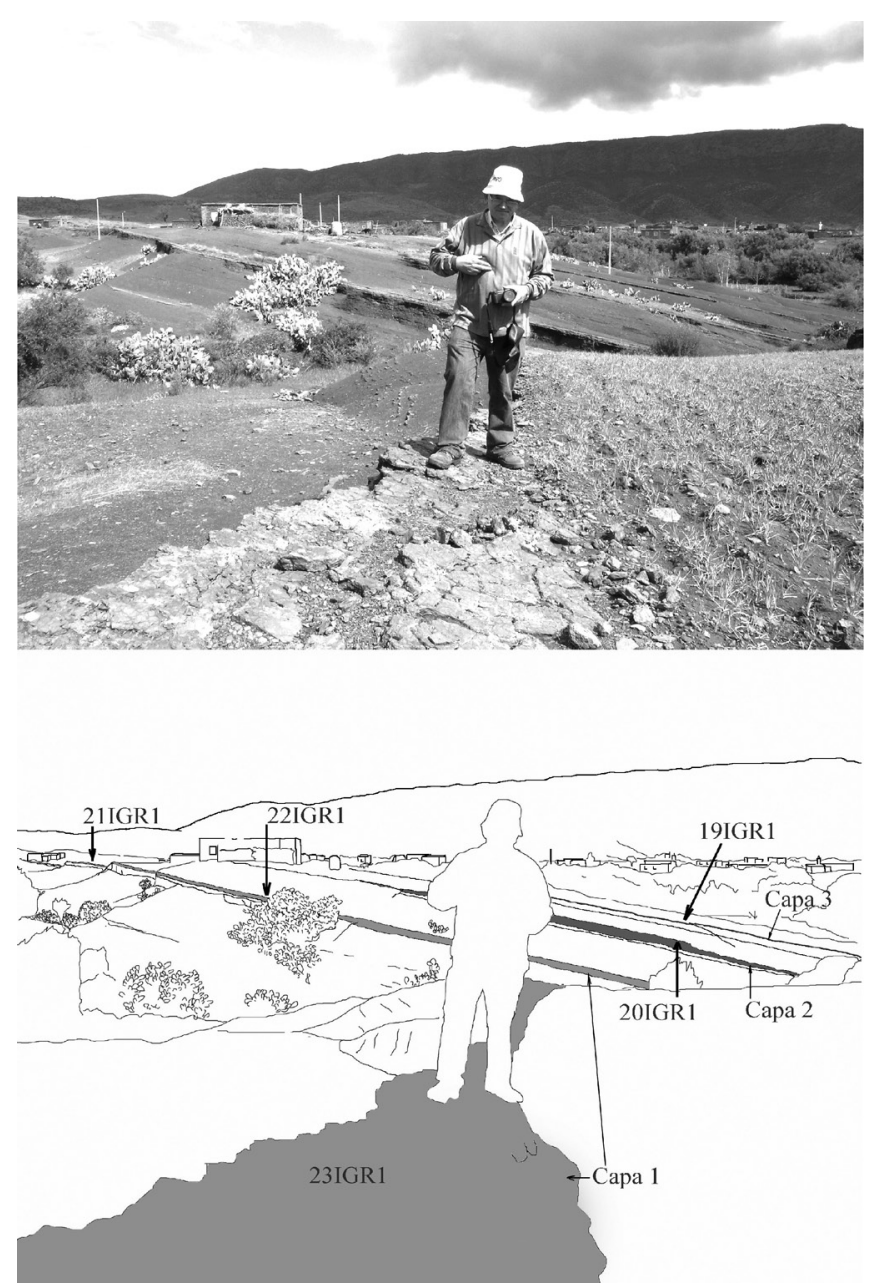

Figura 5. Panorámica con la situación de varios yacimientos y de las capas que los contienen.

Panoramic view of some sites situation and the three stratigraphic beds (Capas).
Solo se ha podido medir el paso $(170 \mathrm{~cm})$ en dos de los afloramientos. La zancada (23IGR1) es de $310 \mathrm{~cm}$.

De los datos anteriores se deduce que la rastrillada es terópoda y producida por un dinosaurio grande $(\mathrm{h}=330 \mathrm{~cm})$, pie estrecho $([-\mathrm{l} a \mathrm{a} / \mathrm{a}=0$ '2 $)$, extremidades gruesas $(\mathrm{z} / \mathrm{l}=4)$, que caminaba con andar moderado (velocidad media de la rastrillada inferior = $4,9 \mathrm{Km} / \mathrm{h}$. Tabla 2) que probablemente dejaba un rastro estrecho.

Rastrillada de la capa intermedia (Figs. 2, 3 y 4; Tablas 1 y 3 )

Formada por nueve icnitas repartidas en dos afloramientos. La distancia entre el primer afloramiento y el segundo es de 620 metros (sobre mapa, figuras 2 y 3) (567 según las medidas del GPS). Esta rastrillada incluye la pista D de Ishigaki (1989) que atribuyó a un rastro de manos de saurópodo, recientemente esta pista ha sido reexaminada por Ishigaki \& Matsumoto (2008) quienes la atribuyen a un terópodo (Fig. 9).

La longitud media de las pisadas es de $77 \mathrm{~cm}$ (máximo de $82 \mathrm{~cm}$, dos huellas) mínimo de $70 \mathrm{~cm}$ (tres huellas de la rastrillada 25IGR1). La anchura media es de $65 \mathrm{~cm}$.

No se aprecian marcas de almohadillas ni de uñas. Dedos grandes, largos e independientes. El «talón» es amplio y redondeado. El ángulo interdigital II^III $\left(23^{\circ}\right)$ es menor que el III^IV $\left(27^{\circ}\right)$.

El paso es de $163 \mathrm{~cm}$ (oscila entre 158 y $166 \mathrm{~cm}$ ) y la zancada media de $321 \mathrm{~cm}$. El ángulo de paso es relativamente grande $\left(160^{\circ}\right)$ y la orientación negativa $\left(-2^{\circ}\right)$.

Las huellas se clasifican como icnitas terópodas, y la rastrillada se supone producida por un dinosaurio grande $(\mathrm{h}=337$ $\mathrm{cm})$, de pie estrecho $\left([\mathrm{ll-a}] /{ }_{\mathrm{a}}=0\right.$ ' 16$)$, extremidades gruesas $(\mathrm{z} / \mathrm{l}=$ 4'0), que caminaba con andar moderado $\left(\mathrm{v}=4^{\prime} 6 \mathrm{Km} / \mathrm{h}\right)$ y de rastrillada muy estrecha $(\mathrm{Ar} / \mathrm{a}=0$ '19)

Rastrillada de la capa superior (Figs. 2 y 4; Tablas 1 y 4)

Contiene una rastrillada de tres huellas de las que únicamente la preservación de la central permite el reconocimiento del po- 


\begin{tabular}{|c|c|c|c|c|c|c|c|c|c|c|c|c|c|c|c|c|c|c|c|}
\hline Huellas & 1 & $\mathrm{a}$ & $\mathrm{P}$ & Ap & $\mathrm{Z}$ & II & III & IV & $\begin{array}{ccc}\wedge & 1 \\
\text { II III IV }\end{array}$ & $\mathrm{Ar}$ & $\mathrm{Lr}$ & 0 & $\mathrm{~h}$ & $2 / \mathrm{h}$ & $\mathrm{v}_{1}$ & $\mathrm{v}_{2}$ & 21 & $\stackrel{(1-a) /}{a}$ & $\mathrm{Ar}_{\mathrm{a}}$ \\
\hline 13IGR1.1 & 79 & 53 & & & & 25 & 38 & 25 & 34--32 & & & & 331 & & & & & 0'49 & \\
\hline 16IGR1.4? & 70 & 55 & & & & 36 & 42 & 36 & $1-19$ & & & & 318 & & & & & $0^{\prime} 27$ & \\
\hline - & & & 170 & & & & & & & & & & & & & & & & \\
\hline \multicolumn{20}{|l|}{ 16IGR1.2 } \\
\hline 16IGR1.1 & 80 & 76 & & & & & & & & & & & & & & & & & \\
\hline 21IGR1.2 & & & 170 & & & & & & & & & & & & & & & & \\
\hline 21IGR1.1 & 79 & 66 & & & & & & & $10-31$ & & & & 353 & & & & & 0'24 & \\
\hline 22IGR1.2 & 70 & 55 & & & & & 45 & 31 & ---40 & & & & 318 & & & & & 0'27 & \\
\hline \multicolumn{20}{|l|}{ 22IGR1.1 } \\
\hline 23IGR1.7 & 74 & 62 & & & & 40 & 48 & 47 & $4-21$ & & & & 313 & & & & & $0^{\prime} 13$ & \\
\hline \multicolumn{20}{|l|}{ |-.-------- } \\
\hline \multicolumn{20}{|l|}{ - } \\
\hline \multicolumn{20}{|l|}{\begin{tabular}{|l}
-1 \\
$-\cdots---$ \\
\end{tabular}} \\
\hline \multicolumn{20}{|l|}{ 23IGR1.3 } \\
\hline 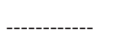 & & & & & 310 & & & & & & & & & 1 & $4^{\prime} 9$ & $4^{\prime} 9$ & $4^{\prime} 2$ & & \\
\hline 23IGR1.1 & & & & & & & & & & & & & & & & & & & \\
\hline
\end{tabular}

Tabla 2. Icnitas de la capa inferior o capa 1 (medidas en $\mathrm{cm}$ ). Abreviaturas usadas en la tabla: 1, longitud de la pisada; a, anchura; P, longitud del paso; Ap, ángulo de paso; z, longitud de la zancada; II, III, IV longitud de los dedos; II^III^IV ángulo entre los dedos; Ar, amplitud de rastrillada; Lr, luz de rastrillada; O, orientación de la huella; h, altura del acetábulo; z/h, zancada relativa; $\mathrm{v}_{1}, \mathrm{v}_{2}$ velocidad $c f$. Alexander/Demathieu; z/l, proporción de la extremidad (esbeltez); (1-a)/a longitud relativa de la pisada; Ar/a, anchura relativa de la rastrillada.

Lower bed footprints or bed 1 (size in cm). Table's abbreviations: $l$, footprint lenght; a, footprint width; $P$, pace length; Ap, pace angulation; $z$, stride length; II, III, IV, digit lengths; II^III^IV, digit angles; Ar, trackway desviation; Lr, trackway width; $O$, orientation; $h$, hip heigth; $z / h$, relative stride length; $v_{1}, v_{2}$ Alexander/Demathieu speed; z/l, legged proportion; (l-a) ${ }_{\text {a }}$ relative footprint length; Ar/a relative trackway width.

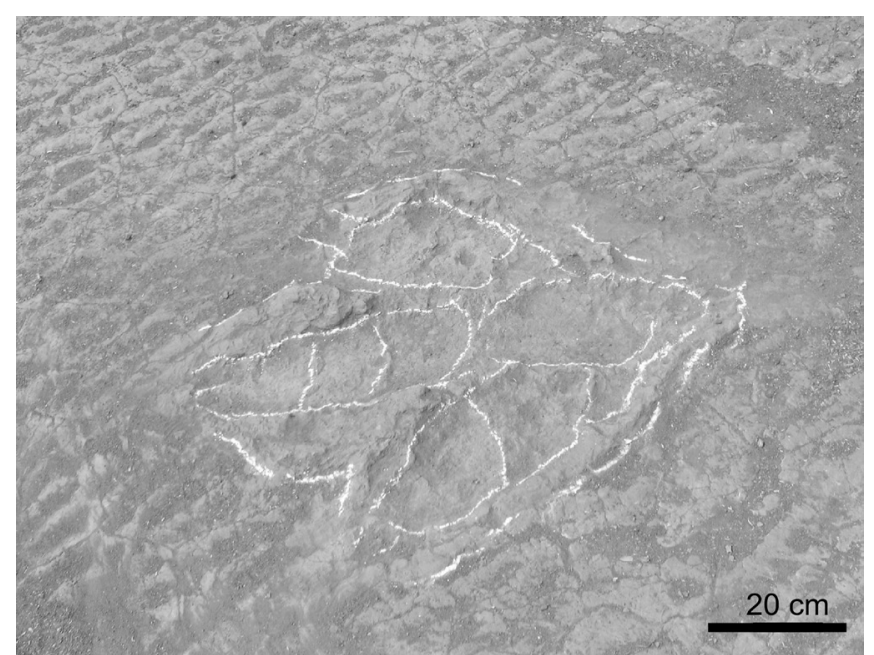

Figura 6. 13IGR1. Marcas de almohadillas y rebabas sobre las estructuras sedimentarias. Grietas de desecación poco patentes.

13IGR1. Digital pads and mud extrusion ridge put on sedimentary structures. Mud cracks not much evidents.

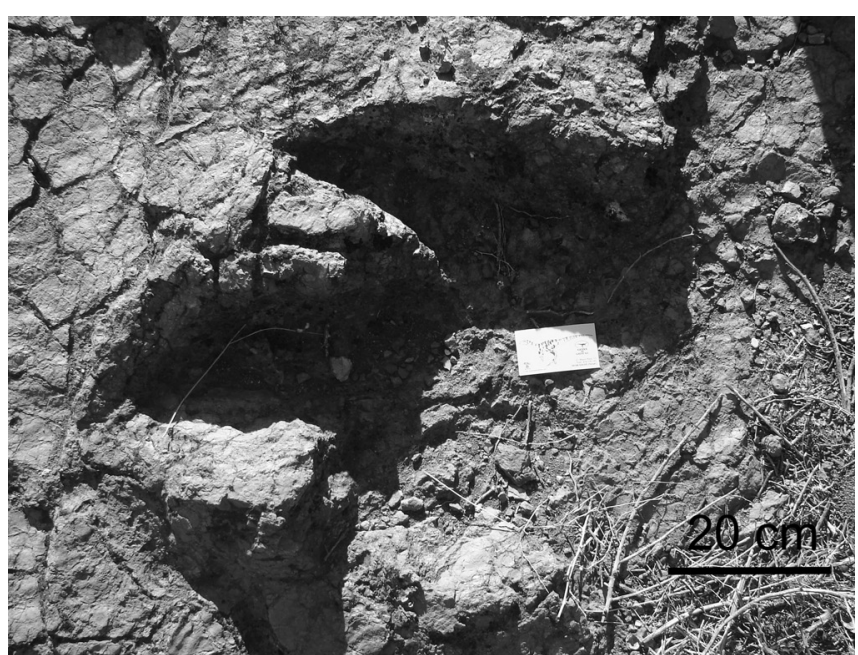

Figura 7. 22IGR1.2 Nivel de grietas de desecación sobre otro de rizaduras. Polígonos de desecación doblados y separados en las rebabas de la huella; rotos en el fondo del hueco de la misma.

22IGR1.2. Mud cracks level on top of a ripple-marks level. Folded and detached dessication polygons in the extrusion ridge, broken in the shaft floor. 


\begin{tabular}{|c|c|c|c|c|c|c|c|c|c|c|c|c|c|c|c|c|c|c|c|}
\hline Huellas & 1 & $\mathrm{a}$ & $\mathrm{P}$ & Ap & $\mathrm{z}$ & II & III & IV & $\begin{array}{c}\wedge \\
\text { II III IV }\end{array}$ & $\mathrm{Ar}$ & $\mathrm{Lr}$ & 0 & $\mathrm{~h}$ & ${ }^{4}$ & $\mathrm{v}_{1}$ & $\mathrm{v}_{2}$ & $4_{1}$ & $(1-\mathrm{a}) / \mathrm{a}$ & $\mathrm{Ar} / \mathrm{a}$ \\
\hline 20IGR1.3 & 80 & 66 & & & & 36 & 45 & 30 & $30-29$ & & & & 334 & & & & & $0^{\prime} 15$ & \\
\hline |--- & & & & & 299 & & & & & & & & & 0'8 & $4 ' 2$ & 4.6 & & & \\
\hline 20IGR1.1 & 81 & 66 & & & & & & & --33 & & & & 338 & & & & & 0'16 & \\
\hline 25IGR1.7 & 77 & 68 & 165 & & & 28 & 48 & 38 & $28-23$ & & & & 345 & & & & & $0^{\prime} 13$ & \\
\hline 25IGR1.6 & 82 & 64 & 158 & 167 & 322 & 34 & 37 & 27 & $9-25$ & 8 & 88 & -7 & 364 & $0^{\prime} 9$ & 4'8 & $4^{\prime} 9$ & $3 ' 9$ & 0 '29 & 0'12 \\
\hline 25IGR1.5 & 70 & 62 & 166 & 158 & 318 & 32 & 45 & 32 & $10-7$ & 15 & 98 & 10 & 318 & $0^{\prime} 9$ & $4 ' 7$ & 4'8 & 4'5 & 0'13 & 0'24 \\
\hline 25IGR1.4 & 70 & 66 & 162 & 156 & 321 & & & & $23-16$ & 22 & 100 & -4 & 318 & $0^{\prime} 9$ & $4^{\prime} 7$ & 4'9 & $4^{\prime} 6$ & 0 '06 & 0'33 \\
\hline 25IGR1.3 & 82 & 64 & 163 & 163 & 321 & 33 & & 23 & $2-34$ & 8 & 92 & -6 & 364 & $0^{\prime} 9$ & $4 ' 7$ & $4^{\prime} 9$ & $3 ' 9$ & 0 '29 & 0'12 \\
\hline 25IGR1.2 & & 62 & 165 & 159 & 323 & & & & $27-34$ & 10 & 97 & -2 & & $0^{\prime} 9$ & 4'8 & $4^{\prime} 9$ & & & 0'16 \\
\hline 25IGR1.1 & 70 & 64 & & & & & & & $23-26$ & & & & 318 & & & & & 0 '09 & \\
\hline
\end{tabular}

Tabla 3. Icnitas de la capa intermedia o capa 2 (medidas en $\mathrm{cm}$ ). Abreviaturas ver tabla 2 . Middle bed footprints or bed 2 (size in $\mathrm{cm}$ ). Abbreviations see table 2.

\begin{tabular}{|c|c|c|c|c|c|c|c|c|c|c|c|c|c|c|c|c|c|c|c|}
\hline Huelllas & 1 & $\mathrm{a}$ & $\mathrm{P}$ & $\mathrm{Ap}$ & $\mathrm{Z}$ & II & III & IV & $\begin{array}{cl}1 \text { A } \\
\text { III II IV }\end{array}$ & $\mathrm{Ar}$ & $\mathrm{Lr}$ & 0 & $\mathrm{~h}$ & ${ }_{4}$ & $\mathrm{v}_{1}$ & $\mathrm{v}_{2}$ & $z_{1}$ & $\stackrel{(1-a) / a}{a}$ & $\mathrm{Ar}_{\mathrm{a}}$ \\
\hline 19IGR1.3 & & & 190 & & & & & & & & & & & & & & & & \\
\hline 19IGR1.2 & 90 & & 180 & 170 & 380 & & & & & 8 & & -14 & 394 & 1 & 52 & $5 ' 4$ & 4 & & \\
\hline 19IGR1.1 & & & & & & & & & & & & & & & & & & & \\
\hline
\end{tabular}

Tabla 4. Icnitas de la capa superior o capa 3 (medidas en $\mathrm{cm}$ ). Abreviaturas ver tabla 2. Upper bed footprints or bed 3 (size in $\mathrm{cm}$ ). Abbreviations see table 2.

sible icnopoyeta. La primera y tercera huella solo se distinguen por una parte de la rebaba posterior y anterior que se conserva.

La icnita intermedia es la más larga de las tridáctilas de la zona $(90 \mathrm{~cm})$. No se puede medir la anchura porque una parte de ella no aflora. La marca del dedo IV está bajo la roca, la del dedo III es redondeada y la del dedo II no es simple y parece que es resultado del movimiento del dedo durante las fases de la formación de la pisada. El paso, ángulo de paso y la zancada son los mayores de los descritos en este trabajo.

La altura de la extremidad se ha deducido para un dinosaurio terópodo. No obstante tal atribución está limitada a las observaciones sobre una sola icnita que además no muestra con claridad la terminación de los dedos.

\section{ESTRUCTURAS SINGENÉTICAS}

\section{Capa inferior/rastrillada inferior}

El techo de la capa inferior presenta rizaduras, sobre las que se han superpuesto grietas de desecación (Figs. 6 y 7). Las pisadas son posteriores a la formación de los polígonos de desecación de barro a los que desplazan, doblan o rompen. En general en las rebabas se ven los polígonos desplazados y doblados y en el fondo del hueco de las huellas suelen estar rotos (Figs. 7 y 8). El desplazamiento de los polígonos en las rebabas es vertical hacia arriba y horizontal centrífugo con respecto a las icnitas. Las grietas de desecación que los separan, se abren más en lugares en los que predomina el desplaza-

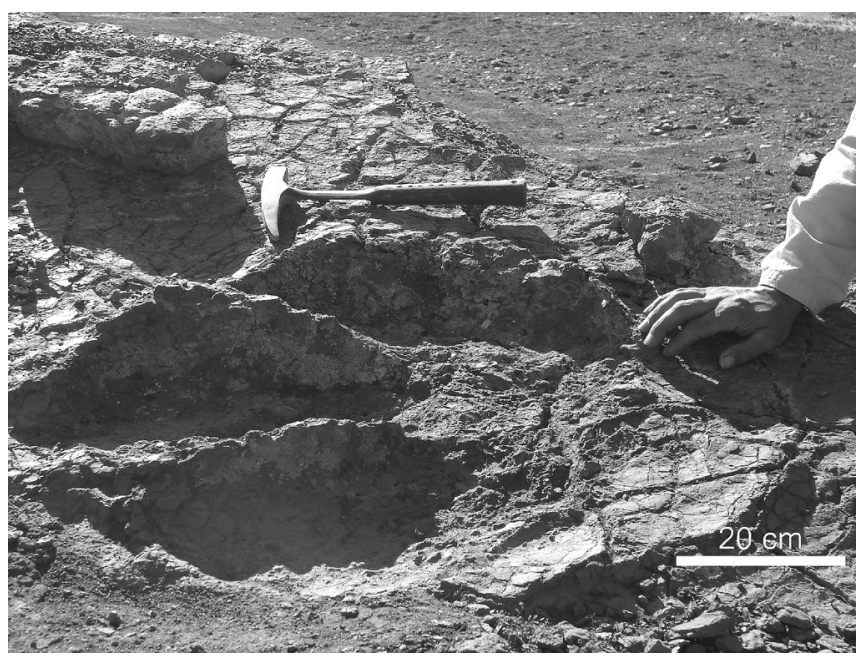

Figura 8. 23IGR1.7. Polígonos de desecación desplazados en la parte trasera y rotos en el fondo de la icnita. Obsérvese la altura de las rebabas.

23IGR1.7. Dessication polygons drifted in footprint rear part and broken in the shaft floor. To observe the high extrusion ridge.

miento vertical y se cierran en donde el desplazamiento es centrífugo.

La separación entre la parte más alta de las rebabas y el fondo del hueco de estas icnitas es relativamente gran- 


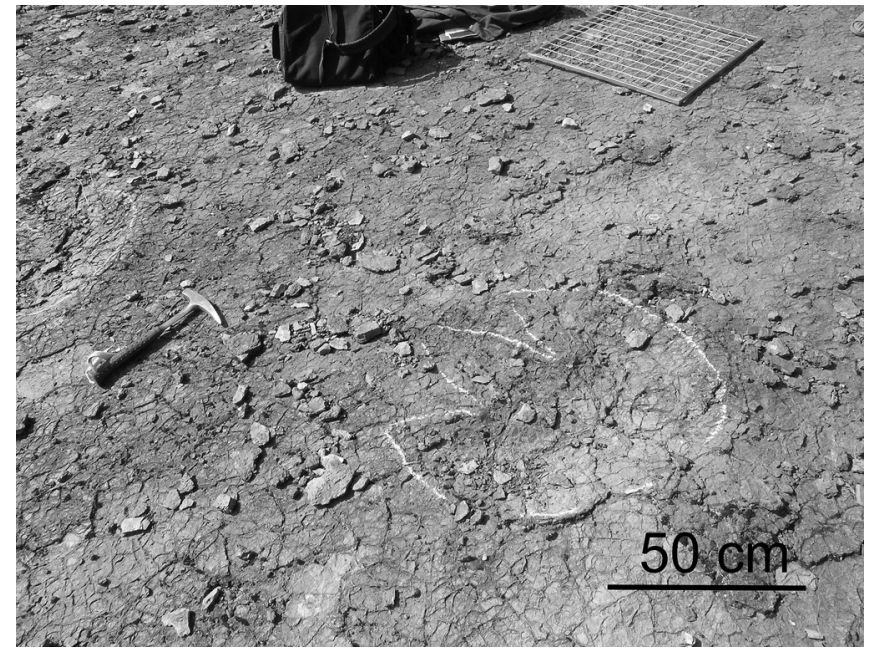

Figura 9. 25IGR12.5 y parte de 25IGR1.6. Polígonos de desecación levemente deformados y desplazados en las rebabas. Rebabas suaves y amplias.

25IGR1.5 and a part of 25IGR1.6. Wide and low extrusion ridge with little deformed and drifted dessication polygons. de (desde 4 a 12 cm; Figs. 7 y 8). Esta medida no siempre se puede tomar porque en ocasiones están rellenas por limolitas sin deformar procedentes de la parte inferior del nivel lutítico suprayacente.

El desplazamiento de los polígonos en las rebabas, su pandeo en las zonas interdigitales y en las paredes de los huecos, y su rotura en el fondo implican que su comportamiento era entre frágil (rotura y desplazamiento de polígonos rígidos) y plástico (pliegues sin rotura) en este proceso, y que deslizaba (fluía) sobre un sustrato todavía más plástico. Es decir que había una capa de barro semiseco con grietas de desecación sobre sustrato de barro fluido.

De los datos anteriores se puede deducir que la superficie de la capa debió ser la superficie de marcha. No se han encontrado ni impresiones de piel, ni estructuras de adherencia del barro a la "planta del pie" por lo que no es totalmente comprobable la suposición enunciada para todas las icnitas de esta capa.

\section{Capa intermedia/rastrillada intermedia}

En los dos afloramientos de la capa intermedia, las icnitas doblan los polígonos de desecación, aunque levemen-

\begin{tabular}{|c|c|c|c|c|c|c|}
\hline número & denominación & longitud & observaciones & edad & localización & citas destacadas \\
\hline 1 & Eutinichnium lusitanicum & 70 & contramolde, hallux y metatarso & Oxfordiense superior & $\begin{array}{l}\text { Portugal (Buarcos, Cabo } \\
\text { Mondego) }\end{array}$ & $\begin{array}{l}\text { Gomes (1915-16) } \\
\text { Henriques (1998) } \\
\end{array}$ \\
\hline 2 & Megalosaurus pombali & 92 & $\begin{array}{l}\text { deformada, sin referencias } \\
\text { suficientes }\end{array}$ & Oxfordiense superior & $\begin{array}{l}\text { Portugal (Pedra da Nau, Cabo } \\
\text { Mondego) }\end{array}$ & Lapparent \& Zbyszewski (1957) \\
\hline 3 & Megalosauripus uzbekistanicus & $72 \times 55$ & $\begin{array}{l}1 \text { imedia? } 72 \mathrm{~cm} \text { en rastrillada de } \\
18 \text { huellas }\end{array}$ & $\begin{array}{l}\text { Oxfordiense sup.- } \\
\text { Kimmeridgiense }\end{array}$ & Uzbequistán-Turkmenistán & $\begin{array}{l}\text { Lockley et al.(1996a, 2000) } \\
\text { Meyer \& Lockley (1997) }\end{array}$ \\
\hline 4 & Megalosauripus & $77 \times 60$ & desaparecidos & $\begin{array}{l}\text { Oxfordiense sup. } \\
\text {-Kimmeridgiense inf. }\end{array}$ & $\begin{array}{l}\text { Portugal (Ribeira do Cavalo, } \\
\text { cantera de Zambujal) }\end{array}$ & $\begin{array}{l}\text { Lokley et al. (2000) } \\
\text { Santos et al.(1995) } \\
\text { Santos (2002) } \\
\end{array}$ \\
\hline 5 & Theropoda & 82 & contramolde & Kimmeridgiense & Asturias & García Ramos et al. (2006) \\
\hline 6 & Theropoda & $90-70$ & & Kimmeridgiense & Marruecos (Iouaridène) & Este trabajo \\
\hline 7 & Megalosauripus & $73 \times 54$ & & Portlandiense & Portugal (Praia do Cavalho) & $\begin{array}{l}\text { Dantas et al.(1994) } \\
\text { Lockley et al.(2000) }\end{array}$ \\
\hline 8 & Theropoda (TR1.3) & 70 & & Portlandiense-Berriasiense & España (La Rioja) & Casanovas et al.(1995) \\
\hline 9 & Buckeburgichnus maximus & 70 & $\begin{array}{l}\text { contramolde, con metatarso y } \\
\text { hallux }\end{array}$ & Berriasiense & Alemania & Kuhn (1958) \\
\hline 10 & Theropoda & 69 & contramolde & Berriasiense & España (Las Villasecas) & Barco et al. (2005) \\
\hline 11 & Theropoda & 100 & $\begin{array}{l}\text { con marca trasera (¿metatarso?), } \\
\text { deformada }\end{array}$ & Albiense sup-Cenomaniense inf. & EE.UU. & Lockley et al. (2006) \\
\hline 12 & Tyrannosauropus petersoni & 81 & $\begin{array}{l}\text { más de un metro de larga en el } \\
\text { dibujo }\end{array}$ & Campaniense & Utah & $\begin{array}{l}\text { Petterson (1924) } \\
\text { Haubold (1971) } \\
\text { Lockely et al.(1983) }\end{array}$ \\
\hline 13 & Theropoda & $74-76$ & una huella & Maastrichtiense & Montana & Manning (2007) \\
\hline 14 & Tyrannosauripus pilmorei & 86 & $\begin{array}{l}\text { contramolde, con metatarso y } \\
\text { hallux }\end{array}$ & Maastrichtiense & Nuevo Méjico & Lockley \& Hunt (1994) \\
\hline 15 & Tyrannosauripus & 80 & calco & Maastrichtiense & Wyoming & $\begin{array}{l}\text { Larson (2003) } \\
\text { Lockley et al.(2003) }\end{array}$ \\
\hline
\end{tabular}

Tabla 5. Las icnitas terópodas mayores (medidas en $\mathrm{cm}$ ). The biggest theropod footprints (size in $\mathrm{cm}$ ). 


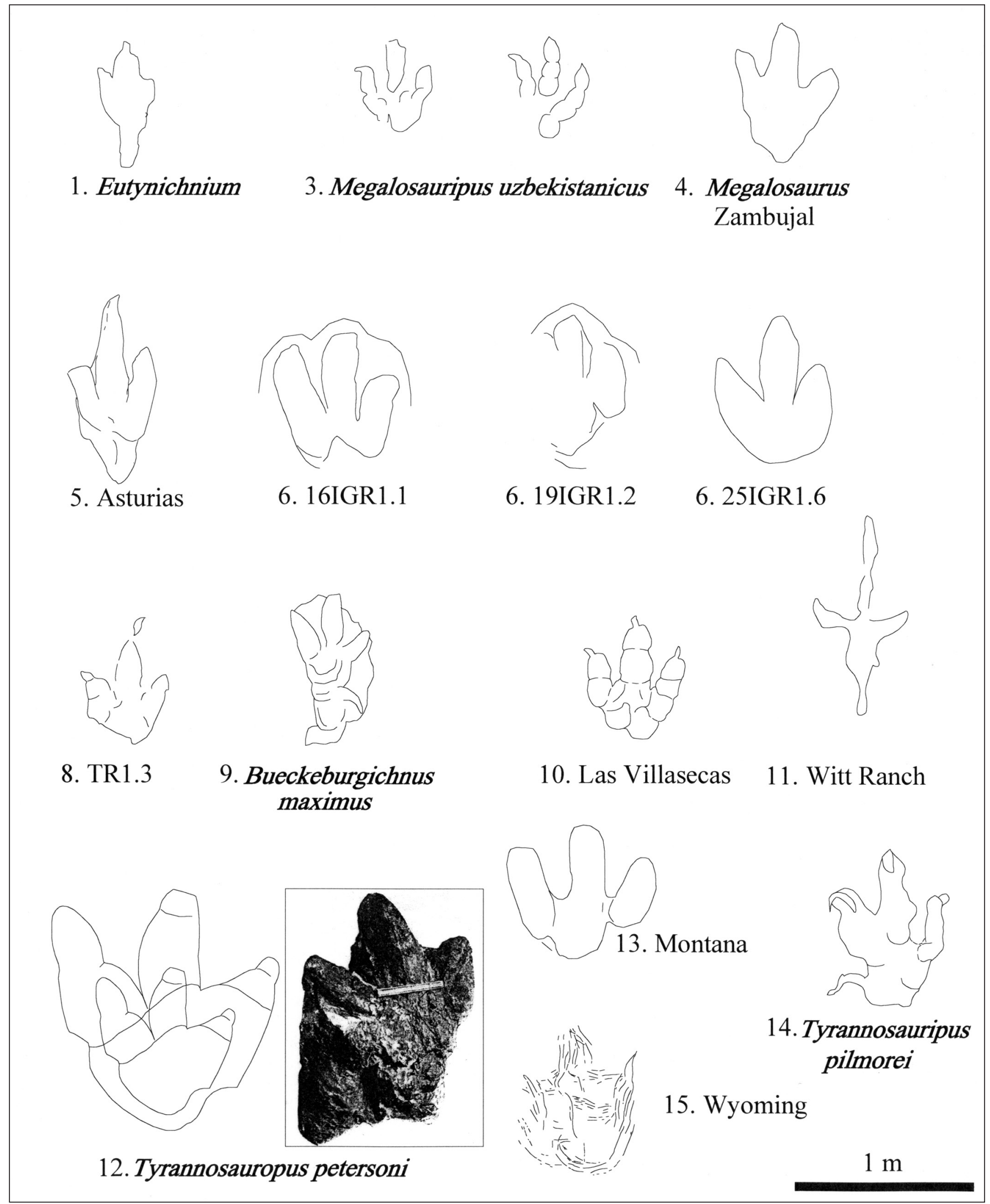

Figura 10. Muestra comparativa de las mayores icnitas terópodas. Redibujado de varios autores (ver texto). El número asignado a las icnitas es el mismo que el de la Tabla 5.

Comparative sampling of the biggest theropod footprints. Redraw from differents authors (see the text). The ichnite number is the same that in the Table 5. 
te. Las rebabas son amplias y muy poco pronunciadas (en general del orden de un centímetro de elevación máxima sobre la capa de estudio). No hay marcas de almohadillas ni de uñas y la línea de contorno, aunque muy aparente, es difícil de precisar (Fig. 9). Es muy probable que estas huellas sean calcos (sensu Romero Molina et al., 2001) muy próximos a la «planta» del pie porque los dedos están bien individualizados.

\section{Capa superior/rastrillada superior}

Las estructuras asociadas a 19IGR1 son parecidas hasta cierto punto a las encontradas en la capa inferior ya que las rebabas de las tres icnitas tienen polígonos de desecación deformados y desplazados. Se diferencian en que en los espacios interdigitales también hay estructuras de flujo del barro. Estas icnitas están claramente en otra capa ya que este estrato concreto no presenta rizaduras, que están presentes en las otras dos capas estudiadas.

\section{ANTECEDENTES SOBRE PISADAS TERÓPODAS MUY GRANDES}

El tamaño de las pisadas (moldes) de dinosaurio oscila entre algo menos de dos centímetros y más de un metro de longitud, estas últimas de dinosaurios saurópodos. Si se consideran solo las icnitas terópodas, la mayor icnita descrita mide $110 \mathrm{~cm}$, y no muestra equivalencia entre tal longitud y la del pie digitígrado que la produjo (Lockley et $a l ., 2006)$, ya que desde su inicio hasta la marca del «talón» hay $65 \mathrm{~cm}$ y desde este hasta la punta más adelantada de los dedos $45 \mathrm{~cm}$ (ver más adelante).

El número de huellas terópodas descritas disminuyte conforme aumenta la longitud de las mismas (Fig. 10). Hay muy pocas que tengan una longintud de más de 65 $\mathrm{cm}$, y las que se acercan o sobrepasan $70 \mathrm{~cm}$ son todavía menos (Tabla 5). En ese grupo, hay varias en las que la longitud de la pisada se ha medido teniendo en cuenta la parte impresa del metatarso. Algunas de las huellas son también contramoldes que pueden medir más que el propio molde (p. ej.: por aplastamiento). Los contramoldes suelen estar producidos por relleno de materiales detríticos de grano mayor (generalmente arenoso) que el material en el que se había formado la impresión de la huella, o más rico en carbonatos. En general, para que se conserve un contramolde, su espesor tiene que ser grande respecto a su longitud y anchura, para que no se parta con facilidad, por lo que, la relación volumen a longitud suele ser alta.

De algunas de las huellas solo existe la referencia escrita, porque se han destruido, y en algunos casos la autenticidad de los datos proporcionados es variable y discutible.

\section{ICNITAS TERÓPODAS DE 70 CM O MAYORES}

\section{a) Con zonas impresas tras la primera almohadilla del dedo IV}

En varias de las huellas la longitud se ha medido incluyendo la marca del metatarso (ver abajo). Si en las icnitas sólo se considera la longitud de la marca de los dedos II-III-IV, es decir sin impresiones de metatarso ni de hallux, varias de las icnitas previamente publicadas como mayores de $70 \mathrm{~cm}$ no alcanzan esta longitud.

1) En Cabo Mondego (Oxfordiense superior, Portugal) hay descritos dos afloramientos con huellas terópodas grandes (Fig. 10; Tabla 5). Las icnitas registradas en ambos yacimientos han recibido distintas denominaciones a lo largo del tiempo: Eutynichnium lusitanicum Nopcsa, 1923 (clasificación seguida por Thulborn, 2001); Megalosaurus pombali Lapparent \& Zbyszewski, 1957; Megalosauripus lusitanicum Lockley, Meyer \& Santos, 1996. Los dos afloramientos citados (Gomes, 1915-1916) eran: Buarcos, que se encontraba en un saliente de roca (huellas en hiporelieve); y de Pedra da Nau, al que se refirieron también Lapparent et al. (1951). Los ejemplares de Buarcos (contramoldes) que se conservan no llegan a 70 $\mathrm{cm}$ (Tabla 5, fila 1) incluyendo la marca del hallux. En la Pedra da Nau Lapparent \& Zbyszewski (1957) hablan de una marca (huella 12c de su dibujo) deformada (Tabla 5, fila 2) de $92 \mathrm{~cm}$ de larga (no hay más referencia de esta impronta y los mismos autores dicen que la talla de las mayores era de 60 a $70 \mathrm{~cm}$, y que una de sus características era la presencia habitual de un cuarto dedo). Si al medir las icnitas de los dos yacimientos no se tiene en cuenta la marca de metatarso y de hallux, la longitud de las pisadas sería del orden de $40 \mathrm{~cm}$. De la huella $12 \mathrm{c}$ solo hay una referencia en un dibujo a mano alzada del yacimiento que no proporciona detalle alguno.

2) En la Cantera de Zambujal en Ribeira do Cavalho (Oxfordiense-Kimmeridgiense inferior, Sesimbra, Portugal) había una pista de dinosaurios terópodos con huellas (Fig. 10.4; Tabla 5) que llegaban a $75 \mathrm{~cm}$ de longitud (Santos, 2002). Una de las huellas dibujada en Lockley et al. (2000) que citan como Megalosauripus mide $77 \mathrm{~cm}$ de longitud y $60 \mathrm{~cm}$ de anchura. Esta icnita era una subhuella en la que también había quedado impresa una parte del metatarso (Santos, 2002). Las huellas de la pista desaparecieron cuando cayó la capa subvertical en la que se encontraban (Santos et al., 1995).

3) En los acantilados de Argüero (Kimmeridgiense, Asturias, España) se han encontrado dos contramoldes grandes ilustrados por medio de dos fotografías por García-Ramos et al. (2006). Las dos huellas son terópodas aunque de morfotipo distinto. Una (Fig. 10.5; Tabla 5) de dedos largos y relativamente delgados de $82 \mathrm{~cm}$ de longitud (op.cit.: 115) y la otra de dedos más cortos, separados e independientes de la que no se dan medidas (op. 
cit.: 122). La icnita de $82 \mathrm{~cm}$ en realidad mide $105 \mathrm{~cm}$ si se considera la marca trasera, probablemente del metatarso. Esta icnita presenta estrías paralelas a la elongación de los dedos visibles en la misma imagen citada, por lo que posiblemente, aunque se trate de una huella real, no es una estampa (sensu Requeta et al., 2006/7). Es el contramolde terópodo más grande citado hasta ahora (Ruiz de Omeñaca, com. pers.)

4) Ballerstedt (1905) describió una icnita de gran tamaño procedente del Berriasiense de Alemania, que posteriormente fue definida como Bückeburgichnus maximus Kuhn, 1958 (ver Fig. 10.9; Tabla 5); el nombre genérico, de acuerdo con la redescripción de Lockley (2000), debe de escribirse Bueckeburgichnus. Thulborn (2001) indica que la redescripción se basó sobre un ejemplar que no correspondía al holotipo. En realidad, el holotipo ( $c f$. Haubold, 1971) tiene $70 \mathrm{~cm}$ de longitud. Estas icnitas muestran la marca de hallux y en la medida de la mayor de ellas (70 $\mathrm{cm})$ también se ha incluido la marca del metatarso. Si se corrige la longitud de la pisada para no tener en cuenta la parte de correspondiente al hallux + metatarso, la longitud de la icnita sería de unos $40 \mathrm{~cm}$.

5) En Witt Ranch (Albiense superior-Cenomaniense inferior, EE.UU.) hay tres huellas (Lockley et al., 2006) muy profundas (hasta $25 \mathrm{~cm}$ ) y muy largas debido a la prolongación trasera de las mismas, probablemente del metatarso (Fig. 10.11; Tabla 5). No se ha hecho el análisis de la dinámica de la formación de la impronta y la interpretación de la prolongación trasera es dudosa porque carecen de impresión del hallux y porque hay estructuras de colapso de barro. Según los autores mencionados la longitud de la pisada 1 es de $87 \mathrm{~cm}$ y de la pisada 2 (que es la que se reproduce en la Fig. 10) de $110 \mathrm{~cm}$. Si se mide la parte digitígrada de las icnitas su longitud sería de 50 y $65 \mathrm{~cm}$ respectivamente.

6) Otra de las icnitas con hallux y con parte impresa por detrás del mismo (Fig. 10.14; Tabla 5) es Tyrannosauripus pilmorei Lockley \& Hunt, 1994 (Maastrichtiense, Nuevo Méjico). Se trata de un contramolde que tiene $85 \mathrm{~cm}$ de longitud, con hallux y metatarso incluidos. Si se resta la medida del sector posterior a la parte proximal de la primera almohadilla del dedo IV, quedan $76 \mathrm{~cm}$ de huella, con lo que sería el segundo contramolde terópodo más grande de la que se conserva una réplica depositada en museo.

\section{b) sin zonas impresas tras la primera almohadilla del dedo IV}

\section{$\underline{\text { Aisladas }}$}

1) Larson (2003) y Lockley et al. (2003) describen un calco de aspecto irregular, procedente del Maastrichtiense de Wyoming (EE.UU.), en el que incluso pueden coincidir la huella de varias pisadas, que asignan a Tyrannosauripus y mide $80 \mathrm{~cm}$ (Fig. 10.15; Tabla 5).
2) Barco et al. (2005) describieron una icnita Berriasiense (Fig. 10.10; Tabla 5) procedente de Las Villasecas (Soria, España). Se trata de un contramolde; la icnita es mesaxónica, pie izquierdo; dedos gruesos con marcas de almohadillas; terminaciones dactilares redondeadas de las que salen uñas estrechas (5,5 a $7 \mathrm{~cm}$ de largas); en el dedo IV se imprimen 4 almohadillas; en el dedo III 2 almohadillas y la parte que se eleva en la unión más alta con el metatarso; dos almohadillas en el dedo II; y finalmente una almohadilla proximal al lado y algo más avanzada que la primera almohadilla del dedo IV; la posición de los dedos es la clásica, con el dedo II separado, el dedo IV cuya parte proximal produce el «talón» de la pisada, y con ángulo interdigital II^III menor que III^IV; huella bilobulada. Es un contramolde de $69 \mathrm{~cm}$ - que aunque no llega a los 70 cm elegidos como dimensión mínima para ser considerados en este estudio- es el que más se aproxima de todos los contramoldes descritos.

3) Manning (2007) realizó una descripción preliminar de una huella de edad Maastrichtiense (Fig. 10.13; Tabla 5), procedente de Montana (EE.UU.); esta huella tiene una longitud de 74-76 cm; dedos anchos y separados; la terminación de las marcas de los dedos no es acuminada. Destaca la asimetría de la huella (dedo II separado y subparelelo al III, dedo IV retrasado y formando ángulo abierto con III). Los dedos tienen terminación redondeada y parecen estar formados por una sola almohadilla. Manning ( $O p$. cit.) propone como posible icnopoyeta a Tyrannosaurus rex; de todos modos hay que esperar a que se realice un estudio más detallado y se asigne de manera precisa esta icnita a un icnotaxon.

\section{Formando parte de rastrilladas}

1) En minas de carbón de Utah y Colorado (Campaniense, EE.UU.) se han citado (Fig. 10.12; Tabla 5) icnitas de 32 pulgadas $(81,28 \mathrm{~cm})$ medidas desde el «talón» hasta la punta del dedo medio por Peterson (1924). Posteriormente, se les describió como Tyrannosauropus petersoni Haubold, 1971, pero se cometió un error en la escala del dibujo de manera que la longitud de la icnita representada (Haubold, 1971: 76, fig. 47, 12) sería de $140 \mathrm{~cm}$. Este mismo error se advierte en la imagen de esta icnita también figurada por Lockley et al. (1983). Se pueden comparar (Fig. 10) el tamaño equivocado y el real y la reproducción de una fotografía de otra icnita gigante del trabajo original en la que se ha respetado la escala - aunque tendrá los errores propios de la perspectiva de la foto. Peterson (1924) muestra una rastrillada de siete huellas en las que señala que la zancada es de $284 \mathrm{~cm}$ y la amplitud de rastrillada de $24 \mathrm{~cm}$. A pesar de que la descripción inicial Peterson (Op. cit.: 390) dice que los dedos son acuminados: "..the toes are so pointed as to indicate the presence of rather sharp claws on the end of each toe". Lockley et al. (1983) interpretan que estas icnitas deberían ser de hadrosaurios; esta afirmación la apoyan tanto en sus obser- 
vaciones actuales sobre las huellas de las minas como en su abundancia, esto último de acuerdo con Peterson (1924: 390) que dice haber encontrado estas huellas en una zona muy extensa "... over an area more than 100 miles in extent and in different seams of coal ..."

2) En la zona fronteriza entre Turkmenistán y Uzbekistán (Oxfordiense-Kimmeridgiense) hay varias rastrilladas terópodas de las que Meyer (1998) menciona que la huella mayor mide $70 \mathrm{~cm}$ de longitud (Fig. 10.3; Tabla 5). Previamente, Amaniyazoz (1985) las había descrito detalladamente como Chodjapilesaurus-Khodzhapilosaurus krimholzi Amaniyazoz, 1985, el cual considera que la longitud media de las huellas es de $48 \mathrm{~cm}$. Lockley et al. (1996b, 2000) indican que las huellas de $\mathrm{Me-}$ galosauropus uzbekistanicus Gabunia \& Kurbatov, 1982 (asignada a Megalosauripus por Lockley et al., 2000), en la rastrillada C llegan a $72 \mathrm{~cm}$ de longitud y 55 de anchura. Aunque en la tabla II del trabajo Lockley et al. (2000) al referirse a Megalosauripus uzbekistanicus (Gabunia \& Kurbatov, 1982) indican esa medida al referirse a la rastrillada $\mathrm{C}$ (que se extinde 26 metros y consta de 18 icnitas), es de suponer que tiene que ser la dimensión de la icnita mayor; ya que la longitud media que indican es de $53 \mathrm{~cm}$. Estas icnitas tienen marcas de almohadillas dactilares (fórmula: 234), pasos de hasta $118 \mathrm{~cm}$, ángulo de paso de $145^{\circ}$ y $212 \mathrm{~cm}$ de zancada (Meyer, 1998). Lockley et al. (2000) dan la longitud de las rastrilladas, de las que la mayor es la número 21 con 311 metros y 141 pisadas, de forma que hasta el momento sería la rastrillada conocida más larga.

3) En Praia do Cavalo (Portlandiense, Portugal), Dantas et al. (1994) describen una rastrillada con 11 icnitas tridáctilas de las que la mayor medía 73 de longitud y 54 $\mathrm{cm}$ de anchura (Tabla 5, fila 7). Los datos medios son 67 $\mathrm{cm}$ de largo por 56 de ancho. El dibujo que se repite en todas publicaciones no proporciona datos suficientes para examinar detalles de la icnita mayor del mismo. Según Santos (2002) la zona está muy meteorizada.

4) En los materiales del Portlandiense-Berriasiense de Treguajantes (La Rioja, España) hay un grupo de huellas terópodas grandes, una rastrillada y una icnita aislada, de las que dos miden más de $67 \mathrm{~cm}$ y otras dos (Fig. 10.8; Tabla 5) más de $70 \mathrm{~cm}$ (Casanovas et al., 1995). Son huellas de dedos anchos e independientes, con marcas claras de más de una almohadilla en cada uno de ellos, y de uñas relativamente largas. La asimetría de los dedos, sobre todo en su terminación trasera es también muy patente (la parte proximal del dedo IV da la zona más atrasada del "talón"). Las huellas son poco profundas y el fondo de la marca de las almohadillas es plano. Barco et al. (2005) asocian este morfotipo con el de Las Villasecas comentado en el apartado anterior (Fig. 10.10).

\section{DISCUSIÓN}

\section{TAMAÑO}

En este trabajo se consideran como huellas digitígradas gigantes aquellas que poseen una longitud mínima de $70 \mathrm{~cm}$. La elección de esta medida hace referencia a que son aquellas que presentan una dimensión similar a las huellas terópodas de Iouaridène que se estudian en este trabajo (Fig. 10; Tabla 5).

Una cuestión de procedimiento es que el tamaño medido en las huellas terópodas de Iouaridène se ha medido sin incluir el hallux ni ninguna marca trasera, por lo que se han seleccionado para la comparativa sólo aquellas huellas que tengan $70 \mathrm{~cm}$ de longitud sin incluir estas estructuras; por lo que no se comparan: Eutynichnium lusitanicum Nopsa, 1923, Bueckeburgichnus maximus Kuhn, 1958, terópodo de Las Villasecas, terópodo de Witt Ranch y Tyrannosauripus pilmorei Lockley \& Hunt, 1994 (Fig.10.1, 10.9, 10.10, 10.11, 10.14; Tabla 5, filas 1, 9, 19, 11, 14).

Las rastrilladas en las que la longitud media de la pisada es inferior a $70 \mathrm{~cm}$, son también de autópodos menores. Entre ellas se incluyen: Megalosauripus uzbekistanicus Lockley, Meyer \& Santos, 2000, Megalosauripus de la Praia do Cavalho y Theropoda (TR1.3) de La Rioja (Fig. 10.3, 10.7, 10.8; Tabla 5, filas 3, 7, 8).

Para que la comparación, desde el punto de vista de preservación, sea coherente tampoco se han considerado aquellas icnitas que se ha constatado que no son estampas, es decir las deformadas, los contramoldes, calcos y las subhuellas. Aunque ciertos contramoldes proporcionan mejor información que ciertas estampas, su análisis queda fuera de este estudio por las particularidades que presentan, distintas al examen de los huecos (sensu Allen, 1997). Huellas en las que se reconocen las especificaciones anteriores son: Megalosaurus pombali Lapparent \& Zbyscewski, 1957, terópodo de Asturias, Tyrannosauropus petersoni Haubold, 1971, Tyrannosauripus pilmorei y Tyrannosauripus de Wyoming (Fig. 10.2, 10.5, 10.12, 10.14, 10.15; Tabla 5, filas 2, 5, 12, 14, 15).

Por otro lado, las icnitas aisladas no permiten obtener los datos promedio: terópoda de Asturias, terópoda de Las Villasecas, terópoda de Montana, Tyrannosauripus pilmorei y Tyrannosauripus de Wyoming (Fig. 10.5, 10.10, 10.12, 10.13, 10.14, 10.15; Tabla 5, filas 5, 10, 12, 13, 14, 15).

Por último, hay algunos casos donde las icnitas han sido destruidas, como el Megalosauripus de Zambujal (Fig. 10.4; Tabla 5, fila 4), por lo que ya no se pueden comparar.

Como queda reflejado en los párrafos anteriores muchas de las icnitas que se han mencionado, una vez aplicados los criterios expuestos no se se pueden considerar utilizables para la comparación que se propone.

Las icnitas terópodas gigantes de Iouaridène tienen de longitud media más de $75 \mathrm{~cm}$ (la huella mayor es de 80 y la menor de $70 \mathrm{~cm}$, rastrillada inferior) y $77 \mathrm{~cm}(82$ y $70 \mathrm{~cm}$, 
rastrillada intermedia) para las dos rastrilladas inferiores. En la rastrillada superior solo hay una huella en la que se puedan hacer medidas y su longitud es de $90 \mathrm{~cm}$. En la capa inferior hay cinco yacimientos (con un total de once icnitas reales), en el intermedio dos (con nueve icnitas que posiblemente sean calcos) y en el superior una (tres icnitas reales).

\section{ICNOTAXONOMÍA}

Las huellas terópodas gigantes generalmente se han atribuido a los icnogéneros Eubrontes Hitchcock, 1845, Gigandipus Hitchcock, 1855, Irenesauripus Sternberg, 1932, Bueckeburgichnus Kuhn, 1958, Megalosauropus Colbert \& Merrilees, 1967, Tyrannosauropus Haubold, 1971, Abelichnus Calvo, 1991, Tyrannosauripus Lockley \& Hunt, 1994, y Megalosauripus Lockley, Meyer \& Santos 2000.

De este listado los géneros Gigandipus (Triásico Superior), Abelichnus (Cenomaniense), Tyrannosauripus (Maastrichtiense) y Tyrannosauropus (Campaniense) son característicos de edades distintas a las icnitas aquí estudiadas, por lo que se pueden descartar.

Independientemente de la presencia de hallux, los caracteres biomórficos y morfométricos de las icnitas marroquíes, permiten diferenciarlas de: Irenesauripus y Bueckeburgichnus por que éstas tienen los dedos más estrechos, además el primer icnogénero tiene los ángulos interdigitales mayores; y por su mayor talla de los icnogéneros $\mathrm{Me}$ galosauropus y Eubrontes que son más pequeños.

De la misma edad y sin marcas de hallux y, o metatarso, sólo se han descrito las icnoespecies asignadas al icnogénero Megalosauripus. Estas icnitas son mucho más esbeltas, o dicho de otra manera - relativamente mucho más largas - que las de Iouaridène, en comparación con su anchura y de dedos también más delgados.

Las diferencias temporales, morfológicas y morfométricas sugieren que estas icnitas pertenecen a un icnogénero aún no descrito, pero su modo de preservación impide su descripción.

\section{GIGANTISMO}

El gigantismo (talla de más de seis metros en vertebrados terrestres, véase la página web del Department of Geology-University of Maryland, 2008) es un hecho constatado en los terópodos (Ceratosauria y Tetanurae). Se han establecido diversos criterios para considerar a un terópodo como gigante, así Farlow et al. (2006) consideran formas gigantes a terópodos cuyas huellas superan los $60 \mathrm{~cm}$ de longitud, o según Barco et al. (2005), los terópodos gigantes tienen que tener talla que supere $250 \mathrm{~cm}$ de altura del acetábulo. Estos mismos autores indican que durante el Jurásico Superior, las formas terópodas grandes pertenecerían a las familias: Allosauroidae, Carcharodontosau- ridae, Spinosauridae y Megalosauridae. Esta diversidad, y las corresponientes diferencias morfológicas y etológicas, no permite ajustar los cálculos relativos al tamaño del cuerpo en relación con la longitud de la extremidad de manera unívoca, por lo que la dimensión de los icnopoyetas de Iouaridène es indeterminada.

En el Jurásico Superior en el Atlas (Marruecos, Argelia y Túnez) no hay descrito ningún terópodo gigante. Las formas grandes más próximas en el tiempo se han encontrado en los pisos del tránsito Cretácico Inferior al Superior (Albiense-Cenomaniense).

Las tres rastrilladas descritas en este trabajo, son de dinosaurios bípedos grandes, a los que se les ha calculado la altura de la extremidad (330 a $337 \mathrm{~cm}$ ) con las fórmulas de las ecuaciones alométricas de Thulborn (1990). El resultado con las fórmulas morfométricas es mayor (340 a $441 \mathrm{~cm}$ ). Si se considera que la longitud del cuerpo viene a ser un múltiplo de entre tres y cuatro veces y media la longitud de la extremidad, el tamaño de estos dinosaurios oscilaría entre 10 y 19 metros. Estas dimensiones permiten consideralos como dinosaurios terópodos gigantes.

\section{CONCLUSIONES}

En el sinclinal de Iouaridène se han encontrado icnitas terópodas gigantes en un área delimitada.

Son las icnitas digitígradas terópodas más grandes descritas hasta ahora. Las mayores (16IGR1.19, 25IGR1.3-7, 19IGR1.2) tienen de dimensiones 80,82 y $90 \mathrm{~cm}$ y pertenecen respectivamente a la rastrillada inferior, intermedia y superior.

La media de las pisadas según la rastrillada a la que pertenecen (inferior: 75,5; media: 77; y superior: 90) es también mayor que la media de otras rastrilladas estudiadas hasta ahora.

Las icnitas tienen orientación paralela y se encuentran en el techo de tres estratos que se pueden seguir en el campo con facilidad. Aunque no están en afloramiento continuo, tienen elementos distintivos (rastrilladas alineadas, características biomórficas y morfométricas, estructuras singenéticas con su formación y la impresión de las icnitas en tres estratos diferentes) que permiten proponer la posibilidad de que formen parte de tres únicas rastrilladas.

En el caso de que esta hipótesis fuera cierta, se trataría de las rastrilladas terópodas mayores descritas hasta la fecha, con una longitud medible para la inferior de 507 m (quizá de 2.000 metros) y para la superior de $567 \mathrm{~m}$.

\section{AGRADECIMIENTOS}

Este trabajo se ha hecho dentro del proyecto de cooperación interuniversitaria Universidad de Rabat-Universidad de La Rioja 
A/721/06 de la Agencia Española de Cooperación Internacional.En este proyecto participan también la Fundación Patrimonio Paleontológico y la Association pour la Protèction du Patrimoine Géologuique Marrocain. En el trabajo de campo han participado por una parte los alumnos del Curso de Verano de Demnat 2007, que ha sido posible gracias a la acción del presidente de la Asociación, Sr. Y. Ennadifi y a el equipo de campo de la Fundación Patrimonio Paleontológico (A. Fernández, A. Ortega, J.C. Pereda, E. Requeta, J.L. Sainz, y J. Herrero de Galve) por su trabajo desinteresado. La empresa Maderas Garnica subvenciona la investigación de uno de nosotros (IDM) mediante una beca en la Universidad de La Rioja.

\section{BIBLIOGRAFÍA}

Alexander, R. McN. 1976. Estimates of speeds of dinosaurs. Nature, 261, 129-130.

Allen, J. R. L. 1997. Subfossil mammalian tracks (Flandrian) in the Severn Estuary S.W. Britain: mechanics of formation, preservations and distribution. Philosophical Transactions of the Royal Society of London, B, 352, 481-518.

Amaniyazov, R. M. 1985. Unique Upper Jurassic dinosaurs tracks in Turkmenistan. In: Problemy Osvoeniya Pustyn (Eds. M. Dzhalilov \& V. P. Novikov). Academia de Ciencias de Turkmenistan, Ashkhabad, 2, 23-31 (en ruso).

Ballerstedt, M. 1905. Über Saurienfährten der Wealdenformation Bückeburgs. Naturwissenschaftliche Wochenschrift. Neue Folge, 31, 481-485.

Barco, J. L., Canudo, J. I., Ruiz-Omeñaca, J. I. \& Rubio, J. L. 2005. Evidencia icnológica de un dinosaurio terópodo gigante en el Berriasiense (Cretácico Inferior) de Laurasia (Las Villasecas, Soria, España). Revista Española de Paleontología, 20, 59-71.

Boutakiout, M., Hadri, M., Nouri, J., Díaz-Martínez, I. \& Pérez-Lorente, F. 2009. Prospecciones paleoicnológicas en el sinclinal de Iouaridène (Alto Atlas Marruecos). Cuantificación de yacimientos y de icnitas. Geogaceta, 45, 51-54.

Calvo, O. 1991. Huellas de dinosaurios en la Formación Río Limay (Albiano-Cenomaniano'), Picun Leufu, Provincia de Neuquen, República Argentina. (OrnithischiaSaurischia: Sauropoda-Theropoda). Ameghiniana, 28, 241-258.

Casanovas, M. L., Fernández, A., Pérez-Lorente, F. \& Santafé, J. V. 1995. Un terópodo carnosaurio en el camino a Treguajantes (La Rioja, España). In: Huellas fósiles de dinosaurios de La Rioja: nuevos yacimientos (Coord. F. Pérez-Lorente). Ciencias de la Tierra, 18, 13-14

Charrière, A., Haddoumi, H. \& Mojon, P.-O. 2005. Découverte de Jurassique supérieur et d'un niveau marin du Barrémien dans les «couches rouges» continentales du Haut Atlas central marocain: implications paléogéographiques et structurales. Comptes Rendues Palevol, 4, 385-394.

Colbert, E. H. \& D. Merrilees. 1967. Cretaceous dinosaur footprints from Western Australia. Journal of the Royal Society of Western Australia, 50, 21-25.

Dantas, P., Santos, V. F., Lockley, M. G. \& Meyer, C. A. 1994. Footprint evidence for limping dinosaurs from the Upper Jurassic of Portugal. Gaia, 10, 43-48.
Demathieu, G. 1986. Nouvelles recherches sur la vitesse des vertébrés, auteurs de traces fossiles. Geobios, 19, 327-333.

Department of Geology-University of Maryland. 2008. Dinosaurs: A natural history. Theropoda I: Dinosaur red in tooth and claw. http://www.geol.umd.edu/ tholtz/G104/ lectures/104therop.html

Dutuit, J. M. \& Ouazzou, A. 1980. Découverte d'une piste de Dinosaure sauropode sur le site d'empreintes de Demnat (Haut-Atlas marocain). Mémoires de la Société géologique de France, N.S., 139, 95-102.

Farlow, J. O., Langston, W., Everett, E., Solis, R., Ward, W., Kirkland, B. L., Hovorka, S., Reece, T. L. \& Whitcraft, J. 2006. Texas giants: dinosaurs of the Heritage Museum of the Texas Hill Country. Heritage Museum, Texas Hill Country, 105 pp.

Fornos, J. J., Bromley, R. G., Clemensen, L. B. \& RodriguezPerez, A. 2002. Tracks and trackways of Myotragus balearicus Bate (Artiodactyla, Caprinae) in Pleistocene aeolianites from Mallorca (Balearic Islands, Western Mediterranean). Palaeogeography, Palaeoclimatology, Palaeoecology, 180, 277-313.

Gabunia, L. K. \& Kurbatov, V. V. 1982. Jurassic dinosaur tracks of Tashkurgan (Uzbekistan, S.S.R.). [Huellas de dinosaurios jurásicos de Tashkurgan (Uzbekistan URSS)]. Abstracts and proceedings of the 23 scientific session Academia de Ciencias de la República Socialista Soviética de Georgia, Tibilisi, 20-22. (en ruso).

García-Ramos, J. C., Piñuela, L. \& Lires, J. 2006. Atlas del Jurásico de Asturias. Ed. Nobel, Oviedo, 225 pp.

Gatesy, S. M. 2003. Direct and indirect tracks features: what sediment did a dinosaur touch? Ichnos, 10, 91-98.

Gomes, J. P. 1915-16. Descoberta de rastos de sáurios gigantescos no Jurásico de Cabo Mondego. Comunicaçoes Comision dos Servicios Geologicos de Portugal, IX, 132-134.

Haubold, H. 1971. Ichnia amphibiorum et reptiliorum fossilium. In: Handbuch der Paläoherpetologie (Ed. O. Kuhn), 18, 1-124.

Hitchcock, E. 1845. An attempt to name, classify and describe the animals that made the fossil footmarks of New England. Proceedings of the American Association of Geologists and Naturalists, 6, 23-25.

Hitchcock, E. 1855. Shark remains from the coal formation of Illinois, and bones and tracks from the Connecticut River sandstone. American Journal of Science, (2) XX, 416-417.

Ishigaki, S. 1989. Footprints of swimming sauropods from Morocco. In: Dinosaur tracks and traces (Eds. D. Gillette \& M. G. Lockley). Cambridge University Press, New York, 83-86.

Ishigaki, S. \& Matsumoto, Y. 2008. Reexamination of manusonly and manus-dominant trackways of sauropod dinosaurs from Middle Jurassic of Morocco. In: The Second International Congress on Ichnology (Cracov). Abstract book and the intra-congress field trip guidebook (Ed. A. Uchman). Institute of Geological Sciences of Poland, Varsovia, 55-56.

Jenny, J. 1982. Maquete de la Carte Géologique du Maroc, 399, feuille Azilal. Carte Géologique du Maroc 1:100.000. 
Jenny, J. 1985. Notes et Mémoires du Service Géologique du Maroc, 399, feuille Azilal. Carte Géologique du Maroc 1:100.000.

Jenny, J., Le Marrec, A. \& Monbaron, M. 1981. Les empreintes de pas de dinosauriens dans le Jurassique Moyen du Haut Atlas Central (Maroc): nouveaux gisements et précisions stratigraphiques. Géobios, 14, 427-431.

Kuhn, O., 1958. Die Färthen der vorzeilichten Amphibien und Reptilien. Verlagshaus Meisenbach, Bamberg, 64 pp.

Lapparent, A. F. 1945. Empreintes de pas de Dinosaurien du Maroc, exposées dans la Galerie de Paléobiologie. Bulletin du Muséum National d'Histoire Naturelle de Paris, 2, 268-271.

Lapparent, A. F. \& Zbyszewski,G. 1957. Les dinosauriens de Portugal. Memorias Servicios Geológicos de Portugal, 2, $63 \mathrm{pp}$.

Lapparent, A. F., Zbyszewski, G., Moitinho, F. \& Veiga, O. 1951. Empreintes de pas de dinosauriens dans le Jurassique du Cap Mondego (Portugal). Comptes Rendues Sommaires de la Société Géologique de France, 1951, 251-252.

Larson, P. 2003. An important trackway site from the Lance Formation (Late Cretaceous) of Niobara County, Wyoming, with new information on the behaviour of T. rex. Journal of Vertebrate Paleontology, 23, 70A.

Lockley, M. G. 2000. An emended description of the theropod footprint Bueckeburgichnus maximus KUHN 1958, and its bearing on the Megalosaur tracks debate. Ichnos, 7, 169-181.

Lockley, M. G. \& Hunt, A. P. 1994. A track of the giant theropod dinosaur Tyrannosaurus from close to the Cretaceous/Tertiary boundary, northern New Mexico. Ichnos, 3, 213-218.

Lockley, M. G., Young, B. H. \& Carpenter, K. 1983. Hadrosaur locomotion and herding behavior: evidence from footprints in the Mesaverde Formation, Grand Mesa Coal Field, Colorado. The Mountain Geologist, 20, 5-14.

Lockley, M. G., Meyer, C. A. \& Santos, V. F. 1996a. Megalosauripus, Megalosauropus and the concept of Megalosaur footprints. In: The Continental Jurassic (Ed. M. Morales). Museum of Northern Arizona Bulletin, 60, 113-118.

Lockley, M. G., Meyer, C. A., Schultz-Pittman, R. \& Forney, G. 1996b. Late Jurassic dinosaur tracksites from Central Asia: a preliminary report on the world's longest trackways. Museum of Northern Arizona Bulletin, 60, 137-140.

Lockley, M. G., Hunt, M., Paquette, S., Bilbey, A. \& Hamblin, A. 1998. Dinosaur tracks from the Carmel Formation, northeastern Utah: implications for Middle Jurassic paleoecology. Ichnos, 5, 255-267.

Lockley, M. G., Meyer, C. A. \& Santos, V F. 2000. Megalosauripus and the problematic concept of megalosaurus footprints. In: Aspects of theropod paleobiology, (Eds. P. Pérez Moreno, T. Holtz Jr., J. L. Sanz \& J. Moratalla). Gaia, 15, 313-337.

Lockley, M. G., Nadon, G. \& Currie, P. J. 2003. A diverse dinosaur-bird footprint assemblage from the Lance Formation, Upper Cretaceous, Eastern Wyoming: implications for ichnotaxonomy. Ichnos, 11, 229-249.

Lockley, M. G., Matsukawa, M. \& Witt, D. 2006. Giant theropod tracks from the cretaceous Dakota Group of northeastern New Mexico. In: Late Cretaceous vertebrates from the Western Interior (Eds. S. G. Lucas \& R. M. Sullivan), New Mexico Museum of Natural History and Science Bulletin, 35, 83-87.

Manning, P. 2007. http://www.nhm.ac.uk/about-us/news/2007/ october/news_12515.html

Meyer, C. A. 1998. Jura- und kreidezeitliche Dinosaurierfährten aus Zentral-Asien (Usbekistan, Turkmenistan). Natur und Museum, 128, 393-402.

Meyer, C. A. \& Monbaron, M. 2002. Middle Jurassic dinosaur tracks from Morocco - Facts and fiction. $7^{\text {th }}$ European Workshop on Vertebrate Palaeontology, p. 27.

Nopcsa, F. B. 1923. Die Familien der Reptilien. Forschritte der Geologie und Paläontologie, 2, 1- 210.

Nouri, J. 2007. La paléoichnologie des empreintes de pas des dinosauriens imprimées dans les couches du Jurassique du Haut-Atlas Central. Tesis Universidad Mohamed V, Rabat, 240 pp. (inédito).

Pérez-Lorente, F. 2001. Paleoicnología. Los dinosaurios y sus huellas en La Rioja. Fundación Patrimonio Paleontológico de La Rioja, 227 pp.

Pérez-Lorente, F. \& Herrero Gascón, J. 2007. El movimiento de un dinosaurio deducido de una rastrillada terópoda con estructuras de inmersión de los pies en el barro y de arrastre de cola (Formación Villar del Arzobispo. Galve, Teruel, España). Revista Española de Paleontología, 22, 157-174.

Pérez-Lorente, F., Hadri, M. \& Boutakiout, M. 2006. Primeras icnitas de dinosaurio en el Sur del Alto Atlas Central. Carixiense (Formación de Arhbalou. Goulmima, Marruecos). Geogaceta, 40, 159-162.

Peterson, W. 1924. Dinosaur tracks on the roof coal mines. Natural History, 24, 388-391.

Plateau, H., Giboulet, G. \& Roch, E. 1937. Sur la présence d'empreintes de pas de Dinosauriens dans la région de Demnat (Maroc). Comptes Rendus sommaires de la Société géologique de France, 1937, 241-242.

Requeta, L. E., Hernández Medrano, N. \& Pérez-Lorente, F. 2006/7. La Pellejera: Descripción y aportaciones. Heterocronía y variabilidad de un yacimiento con huellas de dinosaurio de La Rioja. España. Zubía monográfico, 18-19, 21-114.

Romero, M. M., Pérez-Lorente, F. \& Rivas, P. 2001. Estructuras asociadas con huellas de dinosaurio en La Rioja (España). Zubía, 19, 61-96.

Romero, M. M., Pérez-Lorente, F. \& Rivas, P. 2003. Análisis de la parataxonomía utilizada con las huellas de dinosaurio. In: Dinosaurios y otros reptiles mesozoicos en España (Coord. F. Pérez-Lorente). Ciencias de la Tierra. 26, 13-32.

Santos, V. F. 2002. Pistas de dinossáurio no JurásicoCretácico de Portugal. Consideraçoes paleobiológicas e paleoecológicas. Tesis doctoral, Universidad Autónoma de Madrid, 366 pp. (inédito).

Santos, V. F., Carvahlo, A. M. \& Silva, C. M. 1995. A jazida da Pedreira da Ribeira do Cavalo (Sesimbra) ou a história 
das pegadas de dinossáurios que nunca mais poderemos visitar. Al-madan, II série, 4, 175-177.

Sternberg, C. M. 1932. Dinosaur tracks from Peace River, British Columbia. National Museum of Canada, Annual Report, 1930, 59-85.

Thurlborn, T. 1990. Dinosaur tracks. Chapman and Hall, London, 1-140.
Thulborn, T. 2001. History and nomenclature of the theropod dinosaur tracks Bueckeburgichnus and Megalosauripus. Ichnos, 8, 207-222.

Manuscrito recibido: 21 de Mayo, 2008 Manuscrito aceptado: 12 de Febrero, 2009 